\title{
Optimal test current shape for accurate arc characteristic determination
}

\section{Journal Article}

\section{Author(s):}

Walter, Michael M.; Franck, Christian (1)

Publication date:

2014-08

Permanent link:

https://doi.org/10.3929/ethz-b-000088762

Rights / license:

In Copyright - Non-Commercial Use Permitted

Originally published in:

IEEE Transactions on Power Delivery 29(4), https://doi.org/10.1109/TPWRD.2013.2297400 


\section{(C) 2014 IEEE.}

Personal use of this material is permitted. Permission from IEEE must be obtained for all other uses, in any current or future media, including reprinting/republishing this material for advertising or promotional purposes, creating new collective works, for resale or redistribution to servers or lists, or reuse of any copyrighted component of this work in other works."

Digital Object Identifier: 10.1109/TPWRD.2013.2297400 


\title{
Optimal test current shape for accurate arc characteristic determination
}

\author{
Michael M. Walter, Christian M. Franck, Senior Member, IEEE
}

\begin{abstract}
Arcs under high current gradients ( $>50 \mathbf{k A} / \mathbf{m s})$ are created during fault interruption in passive resonant circuits of HVDC circuit breakers. Black-box models have been successfully applied to model the dynamic arc-network interaction for such breakers. This requires accurately known parameters such as arc cooling power $P(g)$ and thermal arc inertia $\tau(g)$ as a function of conductance. Their experimental determination is difficult and requires significant measurement effort. In a previous work it was shown that these parameters can be determined more accurately with step-wise current measurements compared to sinusoidal test currents. In this contribution, the optimal nonsinusoidal current waveform is addressed, which maximizes the accuracy of $\tau$ at a minimum of required experiments. With a novel arbitrary current source complex, step-, slope- and spikecurrent waveforms are applied to a wall-stabilized arc. The best results have been obtained from a gradually increasing current slope of $0.3 \mathbf{~ k A} / \mathbf{m s}$ superimposed with repetitive current spikes of $5-15 \mathbf{k A} / \mathbf{m s}$ slopes. This enables a large number of evaluation points that showed a high accuracy during a single experiment.
\end{abstract}

Index Terms-arc discharges, current-voltage characteristics, high-voltage techniques, circuit breakers, plasmas, HVDC transmission, current supplies.

\section{INTRODUCTION}

Black-box-models are successful in modeling the dynamic arc-network interaction because they can correctly reflect the transient arc voltage for a wide range of current gradients [1], [2], [3], [4]. Small gradients $<1 \mathrm{kA} / \mathrm{ms}$ are typical for nominal conditions $(<3 \mathrm{kA}, 50 \mathrm{~Hz})$ in $\mathrm{AC}$ networks. Moderate current gradients $<15 \mathrm{kA} / \mathrm{ms}$ can occur in AC circuit breakers at terminal fault interruption [5]. In arc chambers of passive resonant HVDC breakers, current gradients $>50 \mathrm{kA} / \mathrm{ms}$ result during current interruption [3], [4], [6]. This is because such breakers superimpose a high frequency resonant current $(1 \ldots 5 \mathrm{kHz})$ to the fault current in order to create artificial current zero crossings.

Simulation of transient arc-network interaction by black-box models requires accurately known arc characteristics. These are the stationary arc cooling power $P(g)$ and the time of thermal inertia $\tau(g)$, which both vary with conductance $g$ [1], [2], [3]. Their experimental determination is challenging and requires large measurement effort due to the following reasons: The arc undergoes statistical fluctuations because arc chamber parameters are never kept perfectly stable during the interval of measurement [7]. $P$ and $\tau$ affect the transient arc voltage simultaneously and can only be determined independently from each others in a few instances [8]. The required high

M. M. Walter and C. M. Franck are with the Power Systems and High Voltage Laboratories, ETH Zurich, 8092 Zurich, Switzerland, michael.walter@alumni.ethz.ch and cfranck@ethz.ch

Manuscript submitted May, 2013. current waveforms are challenging to create because they must produce arc conductances in a wide range and various current gradients in a single experiment. In a previous work [2], it was shown that certain non-sinusoidal current shapes enable a significantly more precise parameter evaluation compared to classical methods based on sinusoidal current shapes. In this contribution, the optimal non-sinusoidal current waveform for $P$ and $\tau$ determination is addressed, which maximizes the accuracy of $\tau$ at a minimum of required experiments.

The paper is structured as follows: Section 2 addresses the arc parameter determination for black-box models theoretically. In sections 3-6, experiments with a wall-stabilized arc under various current waveforms are presented. Therein, section 3 illustrates the measurement setup, section 4 shows the measured current and voltage waveforms and section 5 discusses the extracted arc parameters. Section 6 concludes this paper.

\section{THEORY}

\section{A. Arc behavior}

An arc is inevitably always exposed to variations of physical conditions. Such variations include arc elongation, electromagnetic deflection, arc root movement and additional ablation when the arc approaches a limiting wall. As a consequence, a measured arc voltage shows continuously stochastic fluctuations for applied currents [7]. This is the case during constant current sections as well as during current slopes. In the former case, the fluctuations lead to stochastically distributed voltage fluctuations around a constant mean value with voltage spikes of varying amplitude and frequency [9].

A vertical wall-stabilized but not convection dominated arc in a narrow nozzle allows - better than any other arcs - to maintain external conditions constant. This is because the flow of heated gas and material evaporated from the nozzle wall stabilizes the arc column. As a consequence, fluctuations are smaller and repetitive experiments become more comparable. In stationary state, the arc is characterized by its mean cooling power $P(g)$, which is typically plotted as $U(I)$ with $U=\sqrt{(P / G)}$ and $I=\sqrt{(P \cdot G)}$. Formerly measured wall-stabilized arcs show falling voltage with current (falling region) at low currents $<200 \mathrm{~A}$ and rising voltage with current (rising region) at high currents. The falling region is explained by a strong increase in ionization with temperature at low currents, causing an increase in conductance [10]. At high currents, ionization saturates and other processes dominate the characteristics. Jenista [11] explains the rising region by a further increase in temperature that causes a strong increase in radiation losses $P \propto T^{4}$, but no additional ionization. As 


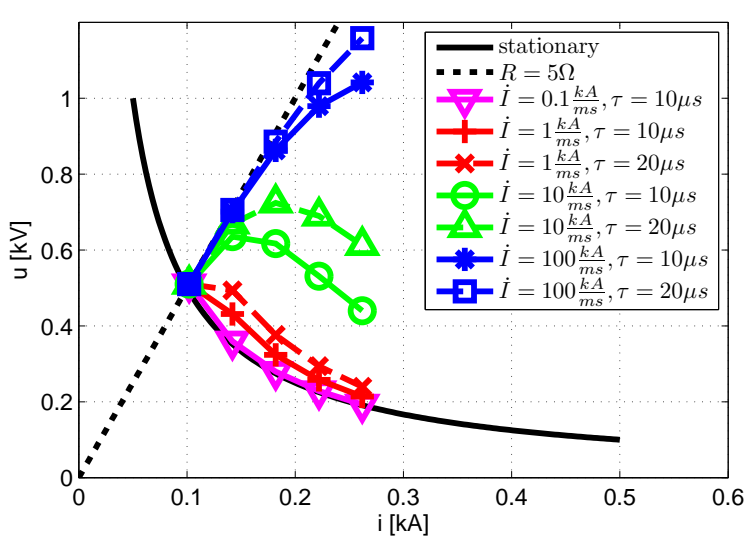

Fig. 1. Effect of thermal inertia at various current gradients

a consequence, the voltage increases moderately with current in the rising region.

\section{B. Black-box modeling}

Black-box models simulate a dynamic arc-network interaction through a single energy balance equation. Thereby they neglect any kind of statistical arc fluctuations. For an applied current, they accurately predict the average stationary arc voltage and all transient overvoltages that are created by an abrupt change in current. Mayr [10] introduced the most commonly used energy balance equation (1), with constant arc power cooling $P$ and time of thermal inertia $\tau$ :

$$
\dot{g}=\frac{1}{\tau} \cdot\left(\frac{I^{2}}{P}-g\right) .
$$

Several experimental studies on various types of arcs have illustrated that parameter functions of the conductance $P(g)$ and $\tau(g)$ describe the arc more precisely than constant parameters [1], [3], [4], [12], [13], [14].

The influence of different current gradients on the transient arc voltage and its consequences for $P$ and $\tau$ determination shall be shortly discussed. For this purpose simple black-box simulations of a wall-stabilized arc are presented with constant arc parameters $P=50 \mathrm{~kW}$ and $\tau=10 \mu$ s and $20 \mu \mathrm{s}$. Fig. 1 shows the resulting $U(I)$ diagram when a current step of $\Delta I=150 \mathrm{~A}$ is applied to an arc burning at a constant current of $I=100 \mathrm{~A}$. Four different slopes $\dot{I}$ are applied to the step: $0.1 \mathrm{kA} / \mathrm{ms}$ (purple), $1 \mathrm{kA} / \mathrm{ms}$ (red), $10 \mathrm{kA} / \mathrm{ms}$ (green), and $100 \mathrm{kA} / \mathrm{ms}$ (blue).

At low current gradients $<0.1 \mathrm{kA} / \mathrm{ms}$, the transient voltage is dominated by the stationary characteristics $P(g)$ because the current rise time $T_{R}=3 \mathrm{~ms}$ is much larger than $\tau$. Under moderate current gradients of $10 \mathrm{kA} / \mathrm{ms}, P$ and $\tau$ affect the transient voltage simultaneously because $T_{R}=30 \mu \mathrm{s}$ lies in the same region as $\tau$. High current gradients of $100 \mathrm{kA} / \mathrm{ms}$ are dominated by the stationary state prior to the current increase. Because of the small rise time $T_{R}=3 \mu$ s the arc does not strongly change its conductance during the current slope, but mainly afterwards. This leads to an ohmic increase in the arc voltage with current during the slope.

\section{Arc parameter determination}

An arc parameter can only be determined from sections of the current-voltage oscillogram where it has a significant effect on the arc voltage. The comparison of transient voltage waveforms with $\tau=10 \mu \mathrm{s}$ and $\tau=20 \mu \mathrm{s}$ reveals that $\tau$ shows the greatest effect around $10 \mathrm{kA} / \mathrm{ms}$ gradients and has no or only a minor effect at gradients $<0.1 \mathrm{kA} / \mathrm{ms}$ and $\geq 100 \mathrm{kA} / \mathrm{ms}$ (cf. Fig. 1). An exception are low gradient slopes or constant currents created subsequent to a high gradient slope [2]. Then, the rate with which the voltage returns to a stationary point is dominated by $\tau$. At low current gradients without previous high gradient slope, the transient arc voltage is closest to its stationary value. Thus, the lower the current gradient is the stronger the influence of the cooling power $P$. We use the terminology direct and indirect to classify the methods [2]: direct, if it does not require a mathematical description of the chosen arc parameters, indirect if it does.

1) Classical determination methods (sinusoidal current): A large number of publications addresses methods for arc parameter determination [8], [12], [13], [14], [15], [16], [17]. They have been compared theoretically [18] and experimentally [2]. All methods were developed to be applied on sinusoidal test current waveforms. This introduces at least one of the following drawbacks: a) The methods rely strongly on the validity of the twice modified Mayr equation because they determine $P$ and $\tau$ simultaneously from a dynamic waveform (e.g. [12], [13], [14], [15], [16], [17]). b) A small number of extraction points (at different conductance) is found, if the evaluation is restricted to extreme points of the sinusoidal waveform, where $P$ and $\tau$ occur decoupled (e.g. [8]). c) Already small fluctuations in the arc voltage (they are not described by the twice modified Mayr equation) can lead to strongly falsified $\tau$ values [2]. d) Indirect determination is used to reduce calculation effort and increase robustness against stochastic fluctuations. To do so, an a priori exponential relationship $P=a \cdot g^{b}$ and $\tau=c \cdot g^{d}$ is assumed and the parameters are not determined directly, but via the coefficients of the chosen function (e.g. [13], [14]). For the characterization of blown arcs in HVAC and HVDC circuit breakers this has been done [1], [3], [13], [14]. However, this relationship may be valid only for certain arcs in a limited range of conductance.

In most methods, a test current must be shaped specifically to produce different conductance gradients over a wide range of conductance. For this purpose, mostly sinusoidal currents are used for arc parameter determination because they are easy to create [8], [12]. Their drawback is the coupling of current amplitude and current gradient $\dot{I}=\omega \cdot \widehat{I} \sin (\omega t)$. As a consequence, only few $P$ and $\tau$ values result per experiment. The number of experiments can be significantly reduced if a high frequent current is superimposed to a low frequency oscillation. Such are: high frequency sinusoidal current superimposed to a $50 \mathrm{~Hz}$ current, high frequency oscillation superimposed to an exponentially decreasing positive current (CR-discharge) [19] or a damped oscillation around current zero (LRC-circuit) [2]. Also passive resonant currents were used for parameter determination [17]. Of these waveforms, none contain constant current sections, and they do not allow 
to vary $I$ and $\dot{I}$ independently from each other.

2) Improved direct determination method (non sinusoidal currents): In a recent publication [2], a series of step currents with high current gradient followed by constant current sections were applied. $P(g)$ was determined from the constant current sections and $\tau(g)$ from the slopes in between. Thereby, a decoupling of the effect of $P$ and $\tau$ was achieved. This allowed the application of a less complex algorithm for parameter determination, by which a smaller scatter of $\tau$ was achieved in comparison to sinusoidal damped currents, for a given number of experiments.

Single $P$ and $g$ values are extracted at two subsequent constant current sections linearized in between. In each current slope, $\tau$ is iteratively fitted to minimize the least square error between the measured conductance gradient

$$
\dot{g}_{m}=\frac{\dot{I} U-\dot{U} I}{U^{2}}
$$

and a prediction $\dot{g}$ by eq. (1). The method has the following advantages over others:

- A voltage response to a step current allows direct verification, if the arc is described by eq. (1). In addition, stochastic fluctuations in the dynamic and transient phase can be identified easily and excluded from the parameter determination.

- The method can be applied for any black-box model equation.

- No previous assumption is made on the shape of $P(g)$ and $\tau(g)$.

- $P$ and $\tau$ are determined independently from each other.

\section{Measurement And Methods}

\section{A. Arc chamber}

A model circuit breaker (cf. Fig. 2) is equipped with two vertically arranged electrodes and an acrylic glass (PMMA) nozzle with a throat length of $L_{T}=50 \mathrm{~mm}$ and cylindrical inner diameter of $d_{T}=20 \mathrm{~mm}$. At both ends, the nozzle widens with an angle of $45^{\circ}$. The fixed lower coppertungsten electrode is of $18 \mathrm{~mm}$ diameter and a movable upper chromium-nickel electrode is of $5 \mathrm{~mm}$ diameter. The lower electrode was fixed at a distance of $L_{i n}=10 \mathrm{~mm}$ below the nozzle, the upper electrode pneumatically moved upwards to a full gap distance of $L_{t o t}=100 \mathrm{~mm}$. Thereby, the main voltage drop occurred inside the nozzle throat. In comparison to that, the voltage drop of the widened nozzle section is negligible. Arc igniting is achieved by applying a small constant current $(<150 \mathrm{~A})$ already $10 \mathrm{~ms}$ before contact separation which is maintained throughout the entire opening period. At full gap width, the desired high current shape is created.

\section{B. Arbitrary current source}

A novel arbitrary current source is used to create complex current waveforms with repetitive constant current sections intermitted by fast current slopes (up to $100 \mathrm{kA} / \mathrm{ms}$ ) in a single experiment [20]. The source consists of three parallel interleaved modules with a performance of $3 \mathrm{kV}$ and $1 \mathrm{kA}$ each (cf. Fig. 3) and is equipped with a common controller. The load

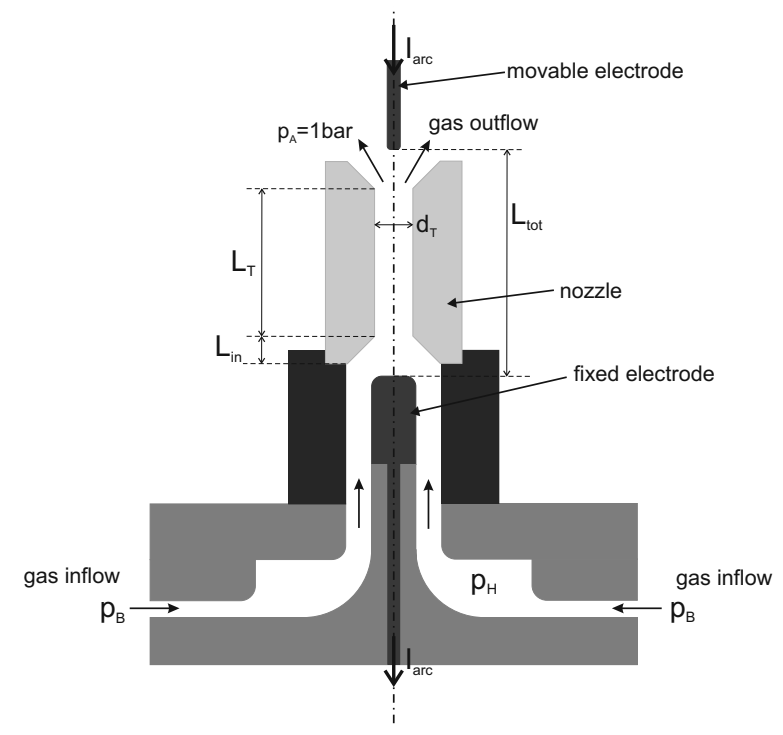

Fig. 2. Schematic plot of the arc chamber.

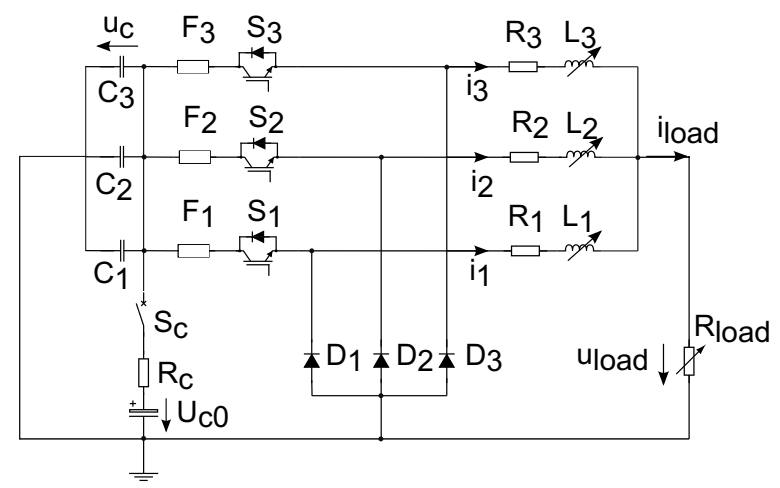

Fig. 3. Electrical equivalent circuit of arbitrary current source

current $i_{\text {load }}$ is the superposition of all three module currents $i_{n}$.

A module consists of a pre-charged capacitor $C_{n}$, an IGBT (insulated-gate bipolar transistor) switch $S_{n}$ with antiparallel diode, an inductor $L_{n}$ and a freewheeling diode $D_{n}$. Each module is protected by a fuse $F_{n}$. The capacitors $C_{n}$ are charged from a DC source $U_{C 0}$ via a charging resistor $R_{C}$. The charging unit $U_{C 0}$ is disconnected during the experiment via $S_{C}$. For closed IGBT a positive voltage difference $u_{C}-u_{\text {load }}$ is applied across the inductor $L_{n}$ and a positive current gradient $\left.\frac{d i_{n}}{d t}\right|_{o n}=\frac{U_{C}-u_{\text {load }}}{L_{n}}$ results. At opened switch, the current commutates to $D_{n}^{L_{n}}$ and the arc voltage $u_{\text {load }}$ causes the current to decrease with a negative gradient $\left.\frac{d i_{n}}{d t}\right|_{o f f}=-\frac{u_{\text {load }}}{L_{n}}$. During a cycle with frequency $f$ and duty cycle $\delta$, the following current step results:

$$
\Delta i_{n}=\frac{u_{C} \cdot \delta-u_{\text {load }}}{L_{n} \cdot f} .
$$

A combination of a micro-controller, a digital signal processor and high speed comparators is used to control the current in each module individually. A file is uploaded before each experiment, containing the three module set-maximal-currents $I_{S n}$ at discrete times plus additional configuration parameters. 
The current in the inductor is monitored via a $5 \mathrm{~m} \Omega$ shunt resistor $R_{n}$ and optically transmitted to the controller. The control cycle frequency $(f \leq 10 \mathrm{kHz})$ must be defined prior to the experiment but can be individually chosen for each discrete time step.

An arc is a very dynamic load that changes its conductance by several orders of magnitude during an experiment. Therefore, a simple but robust pulse-width-modulation (PWM) control strategy was chosen: All switches $S_{n}$ are turned on period-synchronously to $f$ with a selectable phase shift $\varphi_{n}$. An IGBT is turned off immediately when the instantaneous module current $i_{n}$ exceeds the module set current $i_{S n}$ for this time and it remains open until the next control cycle. By that, repetitive experiments become very comparable, no overshoot occurs before a constant current, and no parameter tuning is required when changing the value of a module inductor $L_{n}$.

Prior to each experiment, the size of the inductors $L_{n}$ and the charging voltage $u_{C}$ are selected to achieve the required current gradients $\left(0.1 \mathrm{kA} / \mathrm{ms}<\frac{d i_{n}}{d t}<75 \mathrm{kA} / \mathrm{ms}\right)$ based on a prediction of the arc voltage:

$$
L_{n}=\frac{\left(u_{c}-u_{\text {load }}\right)}{\dot{I}_{n}} .
$$

Each inductor $L_{n}$ is equipped with 10 logarithmically arranged mechanical taps and can be varied in a wide range $(40 \mu \mathrm{H} . .4 .5 \mathrm{mH})$. Complex current waveforms are achieved by cooperation or mutual operation of several modules with different current gradient. An ideal constant current section is achieved by two modules with an inductance ratio

$$
L_{2}=L_{1} \cdot \frac{u_{\text {load }}}{u_{C}-u_{\text {load }}},
$$

so that the positive gradient of one module in on state compensates the negative gradient of the other module in off state.

\section{Adaption of the advanced arc charaterization method}

The new arc characterization method presented in section II-C2 requires that before and after a transient slope a constant current must exist. The following adaption of the method weakens this limit requirement. It determines one stationary point from the transient phase, so that a constant current is required only either before or after the slope.

1) The used current profile has a constant amplitude for a time $t>5 \tau$ to ensure stationary condition. This constant current is followed by a slope with large current gradient. A current waveform can consist of several similar sections at various current amplitudes that fulfill the former condition. If so, the method is applied independently for each section and delivers a single $P$ and $\tau$ value at a single conductance value for each section.

2) Only one stationary value $P_{1}\left(g_{1}\right)$ is determined from the constant current as close as possible to the subsequent current slope.

3) A second stationary value $P_{2}\left(g_{\max }\right)$ is found at the maximum conductance $g_{\max }$ during this experiment. The point $g_{\max }$ is an extremum point $\dot{g}=0$ and is therefore a dynamically occurring stationary point according to Rijanto [8].

4) $\tau$ and $P_{2}\left(g_{\max }\right)$ are determined from a least square fit of eq. (1) to eq. (2).

Current shapes of this kind are for example realized by the superposition of slowly rising currents and current spikes of high gradients. These shapes can be created very efficiently with the present current source by selecting completely different inductances for the different modules. Large inductances for the slowly rising currents and small inductances for those creating the high gradient current spikes.

\section{RESUlts}

A large number of experiments was performed for a wallstabilized arc with identical setup but with different current waveforms applied to the arc. After each measurement series, the nozzle diameter was measured. It widened in more than 150 experiments from $20 \mathrm{~mm}$ to $27 \mathrm{~mm}$. This nozzle widening led to an arc voltage decrease by $\sim 100 \mathrm{~V}$. This effect was also observable in the extracted parameter functions $P(g)$ and $\tau(g)$. A decrease of cooling power $P$ with increasing nozzle diameter and an increase of $\tau$ by a factor 2 from $12 \mu$ s to $22 \mu$ s was observed. The upper electrode length shortened by approximately $0.5 \mathrm{~mm}$ per experiment and was replaced regularly. However, the maximum accepted increase in gap length from 10 to $11 \mathrm{~cm}$ caused only negligible variations in the measurement results.

\section{A. Quasi stationary arc characteristics}

The stationary arc characteristic was investigated for an arc in a $23 \mathrm{~mm}$ diameter nozzle. A slowly rising current gradient of $\dot{I}=+0.35 \mathrm{kA} / \mathrm{ms}$ followed by a falling gradient of $\dot{I}=$ $-0.1 \mathrm{kA} / \mathrm{ms}$ was applied to the arc (cf. Fig. 4a)). The current gradients are small enough so that no transient behavior is excited and the arc follows its quasi stationary characteristics. In the voltage signal, stochastic arc fluctuations of $20-50 \mathrm{~V}$ amplitude and $5-6 \mathrm{kHz}$ are observable. A clearly defined minimum voltage exists to which the arc returns regularly after a fluctuation.

Shown in Fig. 4b) is the quasi stationary $U(I)$ characteristic evaluated from 9 similar low current gradient experiments. Plotted as a function of current are the mean voltage (solid line), the minimum and maximum voltages of all 9 measurements (dashed lines), and the standard deviation of the measurement (error bars). The triangles indicate the average voltages from only the rising slope (pointing upwards) and only the falling slope (pointing down). Variations of up to $25 \mathrm{~V}$ between different experiments can be observed. The standard deviation is $\sim 25 \mathrm{~V}(\leq 10 \%)$ but the difference between minimum and maximum voltage can be as high as $150 \mathrm{~V}$. The mean arc voltage shows the typical falling characteristics at currents below $0.3 \mathrm{kA}$ and a moderate positive voltage increase at high currents. No differences exist between falling and rising current slopes. 

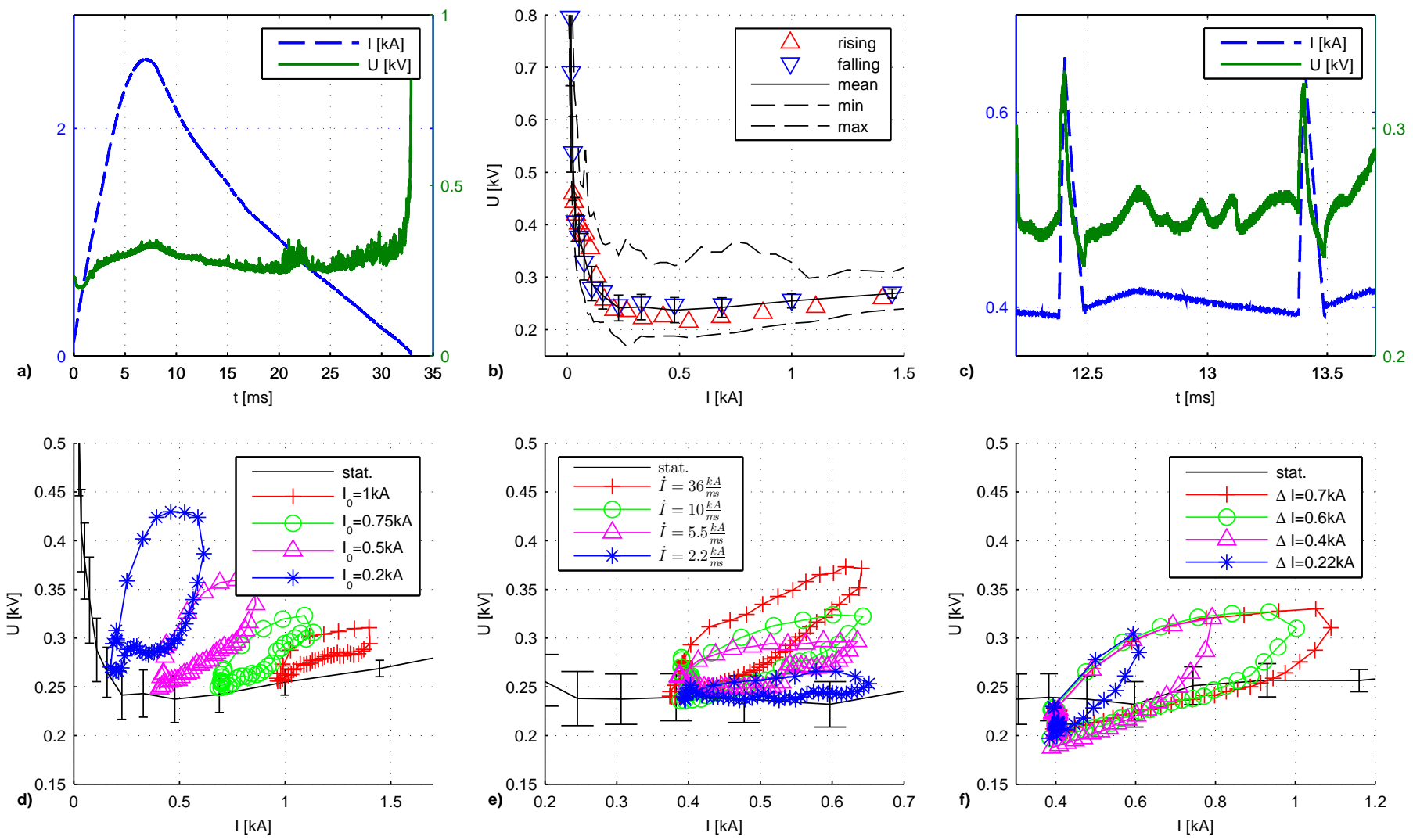

Fig. 4. a): Quasi-stationary arc voltage $U(t)$ during a low gradient current slope, b) $U(I)$-diagram of this experiment (average, min, max, standard deviation), c)-f): Dynamic arc voltage of similar high gradient current spikes of amplitude $\Delta I$ and gradient $I$ subsequent to a constant current of amplitude $I_{0}$, d) Effect of start current amplitude $I_{0}$ at $\dot{I}=36 \mathrm{kA} / \mathrm{ms}$ and $\Delta I=0.5 \mathrm{kA}$, e) Effect of current gradient $\dot{I}$ for a $\Delta I=0.27 \mathrm{kA}$ and $I_{0}=0.4 \mathrm{kA}$, e) Effect of spike amplitude $\Delta I$ for a $\dot{I}=22 \mathrm{kA} / \mathrm{ms}$ and $I_{0}=0.4 \mathrm{kA}$

\section{B. Dynamic arc characteristics}

With the same measurement setup and a nozzle diameter of $24-25 \mathrm{~mm}$, the transient arc voltage during high gradient current spikes was investigated. Rising current slopes are of $\dot{I}=2-36 \mathrm{kA} / \mathrm{ms}$, the falling slopes are lower by a factor 3. A quasi-constant current $I_{0}$ was applied for a time of $0.9 \mathrm{~ms}$ before the spike to ensure quasi stationary conditions. However, the stochastic arc fluctuations cause varying start voltages for all spikes. In the example of Fig. 4c), transient voltage responses of $+100 \ldots-20 \mathrm{~V}$ around the stationary voltage are excited by the current slopes. In comparison to that, stochastic fluctuations are of significantly smaller amplitude.

In the first $\mu$ s of the positive current slope, the arc has not yet started to adopt to the new current value and the effective arc resistance remains constant. The transient overvoltage in this very first period of the spike is thus determined by the start current $I_{0}$ and, resulting from it, the different arc resistances and start voltage. By that, up to a factor 4 higher transient voltages are achieved at low currents in comparison to high start currents (cf. Fig. 4d)). An increase in current gradient $\dot{I}$ reduces the total conductance change during the spike and therefore brings the transient $u(i)$ closer to the initial constant conductance slope (cf. Fig. 4e)). Up to the measured current gradient, this causes a widening of the enclosed area by the transient loop in the $u(i)$ diagram. An increase in spike amplitudes but constant current slope (cf. Fig. 4f)) increases the conductance change during the spike. This deforms the transient $u(i)$ shape, because the parameters $P$ and $\tau$ are not constant for different conductance values. This effect was much stronger observed in the falling region.

\section{Experiment results of step and spike currents}

For arc parameter determination, different current waveforms were applied to a wall-stabilized arc in a $21 \mathrm{~mm}$ nozzle diameter. Only step currents (cf. Fig. 5a)) and spike currents superimposed to a moderately increasing current (cf. Fig. 5b)) are shown here. With the current source step-wise currents with maximally $\dot{I}=16 \mathrm{kA} / \mathrm{ms}$ and a step size of $0.2 \mathrm{kA}$ were applied. The spikes have rising and falling gradients of $29 \mathrm{kA} / \mathrm{ms}$ and $-7 \mathrm{kA} / \mathrm{ms}$, respectively, and their amplitude $\Delta I$ grows with increasing stationary current.

With the new method and its adapted form, the parameter functions $P(g)$ and $\tau(g)$ were extracted for 8 identical step current experiments and 4 identical spike current experiments. Extracted parameters $P(g)$ (respectively $U(I)$ ) and $\tau(g)$ are mostly identical for both current waveforms (cf. Fig. 5c)-e)). From Fig. 5d) it can be seen that in the range of $0.2 \ldots 0.4 \mathrm{kA}$ the stationary arc voltage of the step current exceeds the one from the spike current. This is an artefact originating from the evaluation algorithm. Due to the imperfect step current 

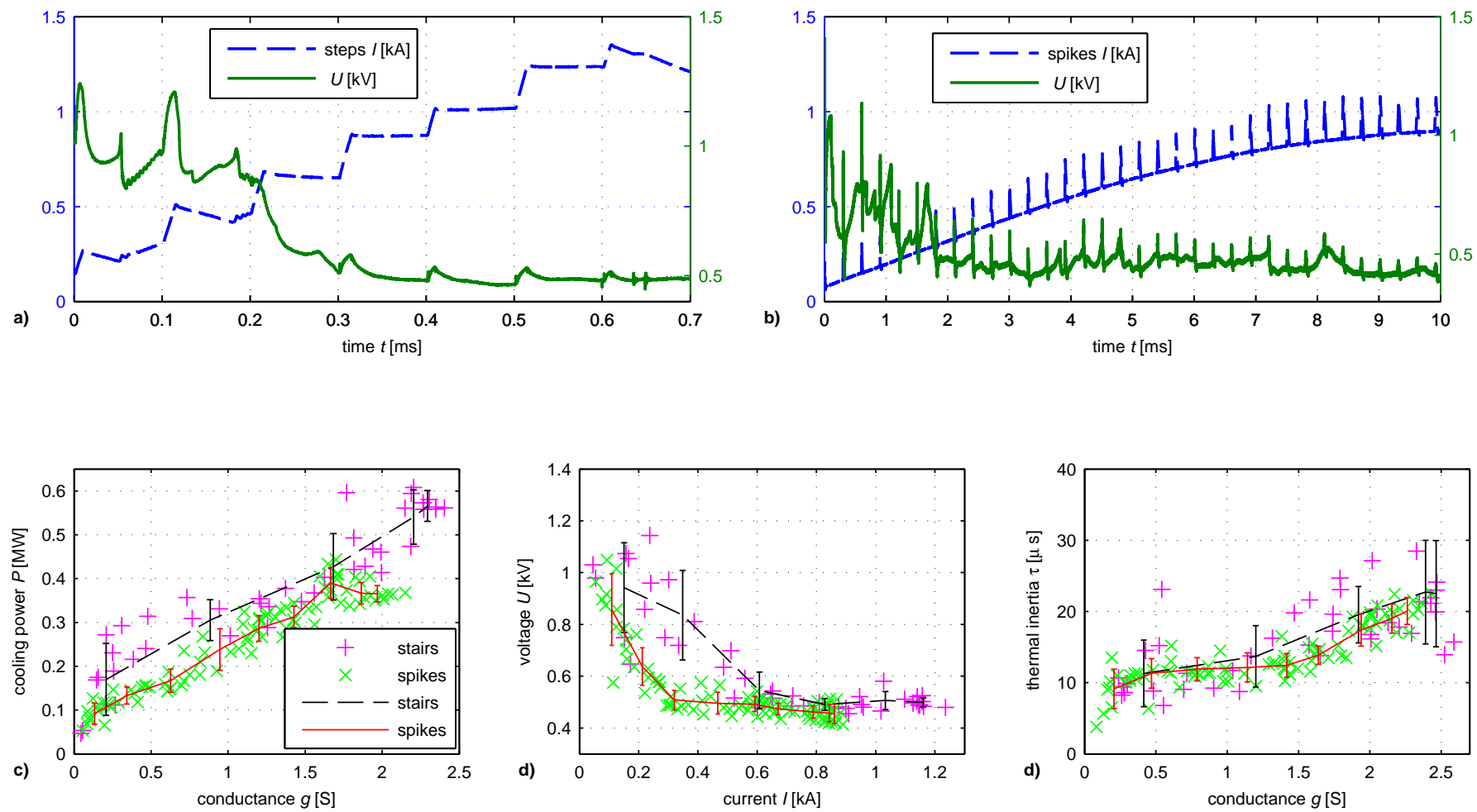

Fig. 5. a) Measured voltage and current during a step-wise test current and b) during a moderately rising current with overlayed spikes. c)-e): derived stationary arc characteristics and thermal inertia.

waveform there is no really constant current section before the step which influences the evaluation. Using the spike current shape it is possible to create four times as many data points as with the step-wise current. Moreover, the scatter in $\tau$ is only $4 \mu \mathrm{s}$ and, thus, a factor of 2 lower.

\section{Effect of current gradient and spike amplitude on param- eter accuracy}

A large number of current spikes as in Fig. 4c) were applied to a $20 \mathrm{~mm}$ wall-stabilized arc to investigate the effect of spike shape on the standard deviation of $\tau$. The influence of current gradient $\dot{I}$ and spike amplitude $\Delta I$ at the falling region $I_{0}=0.15 \mathrm{kA}$ and at the rising region $I=0.4 \mathrm{kA}$ was investigated (cf. Fig. 6). One data point corresponds to four similar experiments with 15-20 identical current spikes each. It can be seen in Fig. 6b) that wrong $\tau$ values result for current gradients $<3 \mathrm{kA} / \mathrm{ms}$, and that the standard deviation decreases significantly towards $25 \mathrm{kA} / \mathrm{ms}$. Fig. 6a) shows that a higher spike current amplitude in the rising region (solid line) leads to a higher $\tau$ value as it is measured over a wider conductance range and consequently at a higher average conduction. In the falling region as well as in the rising region, an optimal spike amplitude exists with the lowest scatter in $\tau$. The optimal amplitude $\Delta I$ has approximately the same value as the quasi stationary current $I_{0}$ before the spike.

With the determined parameters, the transient voltage was re-simulated by an integration of eq. (1) individually for each spike. In Fig. 6c), the average RMS error between the measured voltage and the re-simulated one relative to the difference between maximal and minimal transient voltage occurring during a spike is plotted. A minimal error resulted for an amplitude $\Delta I=0.2 \mathrm{kA}$ in the falling region and an amplitude of $\Delta I=0.3-0.5 \mathrm{kA}$ in the rising region. In both regions, the voltage waveforms could be best recalculated for a gradient of $\dot{I}=15 \mathrm{kA} / \mathrm{ms}$.

\section{Discussion}

Black-box modeling accuracy: For correctly determined arc parameters, the agreement between measured voltage waveform and re-calculation by integration of eq. (1) is very good. Generally, errors $<1 \%$ were achieved. This confirms that the used energy balance equation is suitable to predict transient arc voltages. Larger errors resulted if stochastic fluctuations occurred either in the transient phase or between the instant where the stationary point was determined and the subsequent current slope. Because of the inability to identify very small fluctuations, an average error of $2-4 \%$ resulted for the transient voltage.

Arc fluctuations: In nearly all constant current sections and low gradient slope sections with duration $>100 \mu \mathrm{s}$, stochastic arc fluctuations were present. Up to $20 \%$ of all current steps and spikes showed voltage fluctuations also during the transient phase. Voltage sections that contain such fluctuations are not described correctly by eq. (1) and can lead to wrong $P$ and $\tau$ values. Typically, a too high time constant results. Therefore, the arc must be treated as a stochastic process and the quality of a parameter determination method depends on how well it is possible to exclude or 

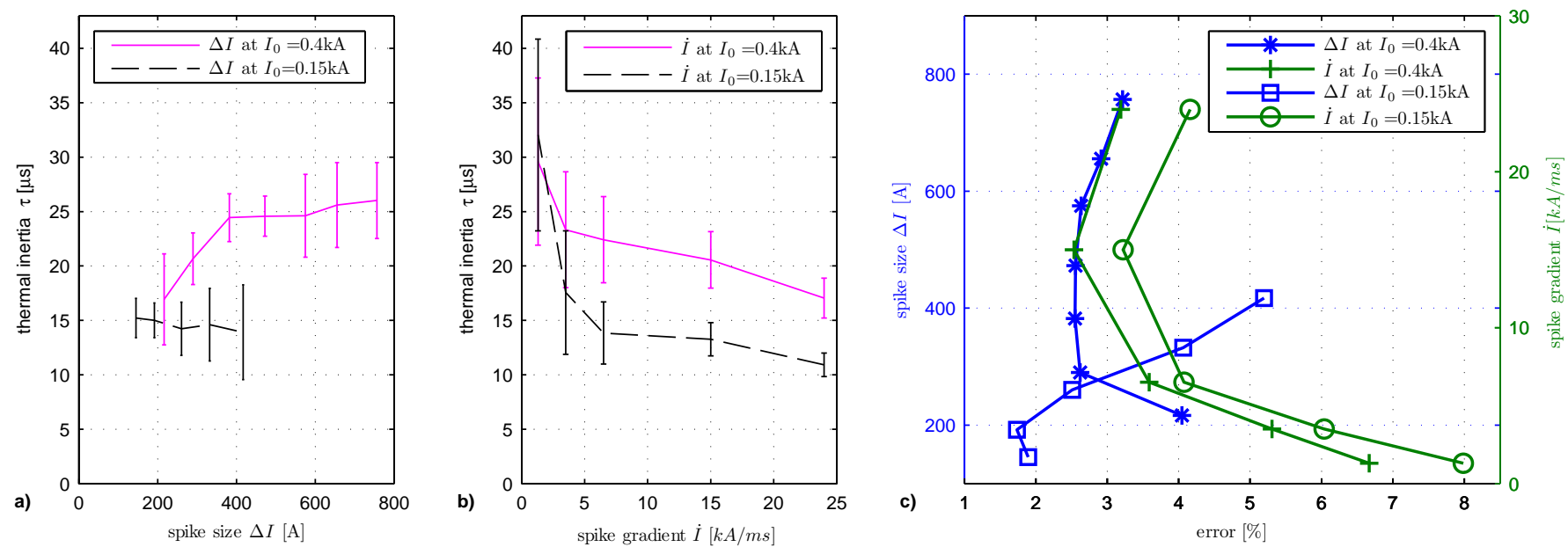

Fig. 6. a) Effect of spike amplitude $\Delta I$ on $\tau$ accuracy for $\dot{I}=25 \mathrm{kA} / \mathrm{ms}$ at falling $(I=0.15 \mathrm{kA})$ and at rising region $(I=0.4 \mathrm{kA})$. b) Effect of spike gradient $\dot{I}$ on $\tau$ accuracy for $\Delta I=0.2 \mathrm{kA}$ in falling region and $\Delta I=0.25 \mathrm{kA}$ in rising region. c) Average error of recalculated transient voltage waveform in $\%$ relative to the difference of maximal and minimal transient voltage.

cope with such fluctuations. A large number of repetitive experiments usually results in a conclusive confidence interval of $P(g)$ and $\tau(g)$. This can be combined with an algorithm to identify large fluctuations and exclude such sections from parameter determination. In the present implementation this was firstly achieved by re-simulation of the voltage waveform and secondly by identification of unexpected maxima and minima in the arc voltage during the constant gradient current slope.

Instantaneous quasi-stationary point: Transient voltages created by current slopes are superimposed to the instantaneous quasi-stationary state and not to an averaged stationary curve. This is in agreement with the observations of [7]. Unless a fluctuation occurs during a current spike, a higher quasi stationary start voltage leads to a constant voltage offset during the whole transient phase. Due to this reason, $\tau$ must be determined with respect to the instantaneous stationary characteristics. This is with higher probability the case, the closer the quasi stationary point is determined to the subsequent current slope, from which $\tau$ is extracted. Thus, Rijanto's [8] approach to merge different gradient slopes from two experiments for parameter determination is likely to cause less accurate $\tau$ values.

Optimal step/spike amplitude: A minimal current increase is required at a given current gradient, so that the transient over-voltage clearly exceeds the arc fluctuations. An upper limit of current step results from the chosen method. The assumption of constant $\tau$ and linearly increasing $P$ during a transient current is not valid if the conductance changes too much during transient currents. To overcome this limitation, the determination algorithm was also implemented in more complex forms including e.g. linearly rising $\tau$, but this too frequently resulted in convergence problems. The conductance variation of a step is typically larger than the one of a spike of similar amplitude, because the spike reaches its maximum conductance value not at current peak but in the falling slope. Therefore, spike amplitudes should be chosen larger than step amplitudes and the difference increases with increasing spike slopes.

Optimal current gradient: The experiments have shown that for spikes, $\tau$ is determined with best accuracy for $\dot{I}=$ $15 \mathrm{kA} / \mathrm{ms}$. At higher gradient spikes, the effect of $\tau$ on the transient voltage decreases because the positive current slope is too short for the conductance to change sufficiently. Also, at higher gradients, the dynamically determined stationary point at $\max (g)$ shows a larger scatter. For steps with very high current gradient, $\tau$ can also be determined from the transient voltage at constant current right after current rise. The second stationary point would then be determined from the same constant current but at a time $>5 \tau$ after the step. This strategy allows the use of significantly higher current gradients and would probably lead to even better accuracy of $\tau$. However, technical constraints of the current source limited the achievable current gradient to $\dot{I}=15 \mathrm{kA} / \mathrm{ms}$ if subsequently a $100 \mu$ s constant current must be created.

Optimal current waveform: Above considerations clearly identify the step currents to be the theoretical optimal current waveform for parameter determination because it completely decouples the determination of $P$ and $\tau$. However, due to the limitation in current gradient, better results were achieved with spikes superimposed to a slowly rising current slope. The fact that transient and stationary behavior of the arc should be determined close to each other demands current waveforms that combine low current gradient sections with high gradient sections. Apart from the proposed waveforms, several other currents would fulfill this condition. Among them Sawtooth currents and high gradient step currents but with significant overshoot have been measured as well. They showed only slightly larger scattering for $\tau$ than the presented measurements.

With the available current source, the optimal current waveforms are spikes because a large number of spikes can easily be created at different amplitudes in a single experiment. For experiments with arcs at several $10 \mathrm{kA}$, such spikes could also 
be superimposed to a $50 \mathrm{~Hz}$ sinusoidal waveform with likely comparable results.

\section{CONCLUSION}

A novel arbitrary current source enabled to create complex current waveforms including step and spike currents. By that, the arc parameters could be determined with a higher accuracy with a lower number of experiments. The arc power loss $P(g)$ is ideally determined from constant current sections. The arc thermal inertia $\tau(g)$ can be determined either from moderate current slopes or at constant currents subsequent to a very steep slope. Therefore, an optimal current waveform combines repetitive constant current sections with high current gradients. This clearly favors a series of small current steps with as large as possible current gradient and constant current section $>5 \tau$. In the experiments, such waveforms were technically limited to a gradient of $15 \mathrm{kA} / \mathrm{ms}$. With this maximum gradient, current spikes superimposed to a current slope with low gradient $(<1 \mathrm{kA} / \mathrm{ms})$ achieved better results. $\tau(g)$ could be determined at 20 different conductance values with a scatter of $<5 \mu$ s from a single experiment. Best results were achieved for spike amplitudes with the size of the previous quasi stationary current. The accurate transient voltage recalculation with error of $2-4 \%$ confirm that it is possible to correctly determine the arc parameters and that the energy balance equation is valid for the investigated wall-stabilized arc.

The improved determination accuracy now allows a better relative comparison of variations in the arc chamber configurations through their black-box parameters. By that, nozzle shape optimization of AC and DC circuit breakers for dynamic processes, such as passive resonance or self-blast pressure build-up, is easier possible.

\section{ACKNOWLEDGMENT}

The work is financially supported by ABB Switzerland Ltd, Corporate Research.

\section{REFERENCES}

[1] CIGRE Report WG 13.01, Applications of black box modeling to circuit breakers, electra No. 149, 1993.

[2] M. Walter, C. M. Franck, Improved method for direct black-box arc parameter determination and model validation, IEEE Trans. Power Del., accepted for publication, 2013.

[3] H. Nakao, et.al., D.C. Current Interruption in HVDC SF6 Gas MRTB by Means of Self-Excited Oscillation Superimposition, IEEE Trans. Power Del., Vol. 16, No.4, 2011.

[4] B. Bachmann, et. al. Development of a $500 \mathrm{kV}$ airblast HVDC circuit breaker, IEEE Trans. Power App. Sys., Vol. 104, No.9, 1985.

[5] CIGRE Report WG A3.23, Application and Feasibility of Fault Current Limiters in Power Systems, ISBN: 978-2-85873-189-3, 2012.

[6] C. M. Franck, HVDC Circuit Breakers: A Review Identifying Future Research Needs, IEEE Trans. Power Del., Vol. 26, No. 2, 2011.

[7] K. Möller, et.al., Theoretical and Experimental Investigation of the Stochastic Behavior of an SF6 Blast-Switching Arc, IEEE Trans. Plasma Science, Vol. 8, 1980.

[8] H. Rijanto, Ein experimentelles Verfahren zur Bestimmung von Lichtbogenkenngrößen - Stromüberlagerungsverfahren, Ph.D. Dissertation, Fakultät für Maschinenwesen, TU Hannover, 1975.

[9] A.D. Stokes, W.T. Oppenlander, Electric arcs in open air, J. Phys. D: Appl. Phys., Vol 24, Jan. 1991.

[10] O. Mayr, Beiträge zur Theorie des statischen und des dynamischen Lichtbogens, Arch. f. El.-Tech., vd. 37, pp 588, Germany, Dec. 1943.
[11] J. Jenista, Static characteristics of a conduction and radiation dominated electric arc in nitrogen, Czechoslovak Journal of Physics, Vol. 43, No. $11,1993$.

[12] W. Rother, Die Rolle des black-box-Modells in der Schalterentwicklung, Intern. Wiss. Koll, TH. Ilmenau, 1980.

[13] J. Schwarz, Berechnung von Schaltvorgängen mit einer zweifach modifizierten Mayr-Gleichung, ETZ-A, bd. 93, pp 386, 1972.

[14] G. Pietsch, H. Rijanto, G. Thiel, Schaltlichtbögen im elektrischen Netz, ETZ-A. Vol. 96, pp. 222-226, 1975.

[15] R. Amsinck, Verfahren zur Ermittlung der das Ausschaltverhalten bestimmender Lichtbogenkenngrößen, ETZ-A, bd. 98, pp. 566, 1977.

[16] H. Drebenstedt, W. Rother, C. Weber, P. Zahlmann, Ein verbessertes Verfahren zur Bestimmung der charakteristischen Funktionen des Zweipolmodells für Schaltlichtbögen, 28. Internat. Wiss. Kolloquium, TH Ilmenau, 1983.

[17] C. Leu, Exitation and course of current chopping oscillation as an intermittent oscillation in SF6, $9^{\text {th }}$ SAP, Lodz, 2001.

[18] B. Spockmann, Auswerteverfahren zur Bestimmung der Kenngröen phänomenologischer Lichtbogenmodelle, Arch. für Elektrotechnik, Vol. 66, 1983.

[19] M. M. Walter, C. M. Franck, Influence of arc chamber parameters on passive resonance circuit of HVDC circuit breakers, CIGRE Session, Bologna, 2011.

[20] M. M. Walter, C. M. Franck Flexible pulsed DC-source for investigation of HVDC circuit breaker arc resistance, Int. Conf. on Gas Discharges and their Applications, Greifswald Germany, pp. 170, 2010.

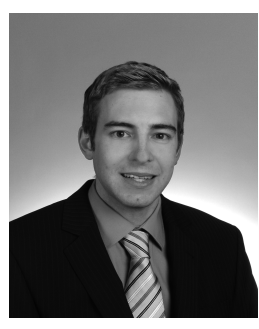

Michael M. Walter was born in Zurich in Switzerland in 1984. He received his Master Degree in Electrical Engineering and Information Technology from ETH- Zurich in 2010. Currently he is researching on Switching arcs in passive resonant HVDC circuit breakers in the scope of his Ph.D. at ETHZurich. His employments for industrial projects and internships during his Master-Studies include the Mitsubishi Electric Corporation in Amagasaki city, Japan, the ABB Switzerland AG (Corporate Research in Dättwil and the ATPT department in Turgi) and the Institute Mihajlo Pupin in Belgrade, Serbia.

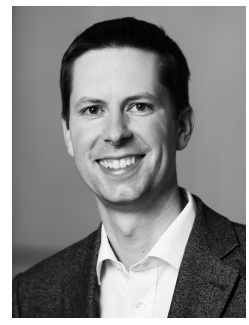

Christian M. Franck (M'04-SM'11) received a diploma in physics from the University of Kiel, Germany in 1999 and the Ph.D. degree in physics from the University of Greifswald, Germany in 2003. He was with the Swiss corporate research center of ABB from 2003-2009 as a Scientist and Group Leader for gas circuit breakers and high-voltage systems. Currently he is an Assistant Professor for High Voltage Technology at the Swiss Federal Institute of Technology (ETH), Zurich, Switzerland. 